\title{
ARTICLE
}

Clinical Study

\section{Whole-colon investigation vs. flexible sigmoidoscopy for suspected colorectal cancer based on presenting symptoms and signs: a multicentre cohort study}

Amanda J. Cross ${ }^{1}$, Kate Wooldrage ${ }^{1}$, Emma C. Robbins ${ }^{1}$, Kevin Pack ${ }^{1}$, Jeremy P. Brown ${ }^{1}$, William Hamilton ${ }^{2}$, Michael R. Thompson ${ }^{3}$, Karen G. Flashman ${ }^{3}$, Steve Halligan ${ }^{4}$, Siwan Thomas-Gibson ${ }^{5}$, Margaret Vance ${ }^{5}$, Brian P. Saunders ${ }^{5}$ and Wendy Atkin ${ }^{1}$

\begin{abstract}
BACKGROUND: Patients with suspected colorectal cancer (CRC) usually undergo colonoscopy. Flexible sigmoidoscopy (FS) may be preferred if proximal cancer risk is low. We investigated which patients could undergo FS alone.

METHODS: Cohort study of 7375 patients ( $\geq 55$ years) referred with suspected CRC to 21 English hospitals (2004-2007), followed using hospital records and cancer registries. We calculated yields and number of needed whole-colon examinations (NNE) to diagnose one cancer by symptoms/signs and subsite. We considered narrow (haemoglobin $<11 \mathrm{~g} / \mathrm{dL}$ men; $<10 \mathrm{~g} / \mathrm{dL}$ women) and broad ( $<13 \mathrm{~g} / \mathrm{dL}$ men; $<12 \mathrm{~g} / \mathrm{dL}$ women) anaemia definitions and iron-deficiency anaemia (IDA).

RESULTS: One hundred and twenty-seven proximal and 429 distal CRCs were diagnosed. A broad anaemia definition identified $80 \%$ of proximal cancers; a narrow definition with IDA identified 39\%. In patients with broad definition anaemia and/or abdominal mass, proximal cancer yield and NNE were 4.8\% (97/2022) and 21. In patients without broad definition anaemia and/or abdominal mass, with rectal bleeding or increased stool frequency (41\% of cohort), proximal cancer yield and NNE were 0.4\% (13/3031) and 234 . CONCLUSION: Most proximal cancers are accompanied by broad definition anaemia. In patients without broad definition anaemia and/or abdominal mass, with rectal bleeding or increased stool frequency, proximal cancer is rare and FS should suffice.
\end{abstract}

British Journal of Cancer (2019) 120:154-164; https://doi.org/10.1038/s41416-018-0335-z

\section{INTRODUCTION}

Patients referred to hospital with suspected colorectal cancer (CRC) typically undergo whole-colon investigation (WCI), predominantly colonoscopy or computed tomography (CT) colonography, in line with National Institute for Health and Care Excellence (NICE) guidelines. ${ }^{1}$ In 2015, NICE issued a guideline on referral criteria, including symptoms and signs conferring a positive predictive value for cancer of $3 \%{ }^{2}$ Consequently, large numbers of patients are undergoing $\mathrm{WCI}$ for suspected $\mathrm{CRC}$, placing pressure on endoscopy and radiology services and incurring substantial costs to the National Health Service (NHS). ${ }^{3,4}$ Reducing the burden of symptomatic referrals on diagnostic services is recognised as a priority area for research. ${ }^{5}$

Flexible sigmoidoscopy is quicker, safer, less complicated, and cheaper than colonoscopy. Intravenous sedation is usually not needed and enemas used for preparation are associated with fewer side effects and greater acceptability than oral preparations used for WCl. $^{6}$ Flexible sigmoidoscopy can be performed competently by non-physician endoscopists ${ }^{7}$ and has high sensitivity for CRCs in the distal colon and rectum ${ }^{8-10}$; however, it can only reach the splenic flexure at best and so abnormalities in the proximal colon are only found if distal findings precipitate subsequent $\mathrm{WCl}$.
Previous research has demonstrated that presenting symptoms/ signs are associated with CRC location..$^{8-14}$ In the largest previous study, Thompson et al. defined a patient subgroup at low risk of proximal cancer, for whom they deemed examination by flexible sigmoidoscopy alone appropriate. ${ }^{8}$ However, subsequent studies reached variable conclusions regarding sole use of flexible sigmoidoscopy in any patient subgroup, ${ }^{9-14}$ and the current NICE guidelines only recommend it for patients with major comorbidity, in association with barium enema. ${ }^{1}$ The aim of the present SOCCER (Symptoms of Colorectal Cancer Evaluation Research) study ${ }^{15}$ was to further investigate whether presenting symptoms/ signs could be used to identify patients at low risk of proximal cancer, for whom flexible sigmoidoscopy would suffice. This would help alleviate the burden of $\mathrm{WCl}$ on patients and endoscopy and radiology services.

\section{METHODS}

Study design and participants

We included patients who had been referred to 1 of the 21 English NHS hospitals from 2004 to 2007 for investigation of symptoms/ signs suggestive of CRC. Of these hospitals, 8 were general and 13

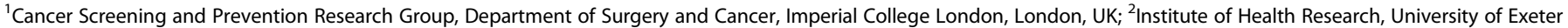

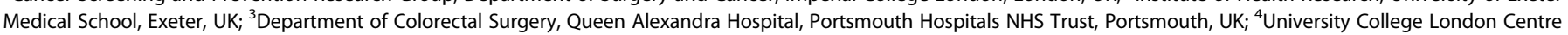
for Medical Imaging, University College London, London, UK and ${ }^{5}$ Wolfson Unit for Endoscopy, St Mark's Hospital, London, UK

Correspondence: Amanda J. Cross (amanda.cross@imperial.ac.uk)

These authors contributed equally: Amanda J Cross, Kate Wooldrage

Received: 11 July 2018 Revised: 17 October 2018 Accepted: 24 October 2018

Published online: 19 December 2018 
were teaching hospitals, and they varied from $<40$ to $>1000$ beds in size.

We retrospectively identified patients from those who were assessed for eligibility for the SIGGAR (Special Interest Group in Gastrointestinal and Abdominal Radiology) trials. The SIGGAR trials were two parallel randomised controlled trials assessing the clinical and cost-effectiveness of CT colonography vs. barium enema and colonoscopy for CRC diagnosis. ${ }^{16,17}$ To be eligible for the SIGGAR trials, patients had to be $\geq 55$ years and judged to be in need of, and fit enough for, a $\mathrm{WCl}$ with full bowel preparation. Patients were ineligible if they were in follow-up for CRC, had undergone $\mathrm{WCl}$ within the previous 6 months, had a known diagnosis of familial adenomatous polyposis or Lynch syndrome, or had previously been diagnosed with inflammatory bowel disease (IBD).

Research nurses undertook the eligibility assessment, checking endoscopy and radiology databases, and patient records and notes. Only patients who were deemed eligible, gave informed consent, and had a consultant consent to their participation were randomised in the SIGGAR trials. Reasons for patient- and consultant-declined consent included patients wanting to have or avoid a specific type of $\mathrm{WCl}$, consultants requesting a specific procedure, and prior cancer diagnoses.

All patients who met the SIGGAR trial eligibility criteria (regardless of whether they proceeded to randomisation) were eligible for the SOCCER study, unless they were judged incapable of giving informed consent, had dissented to use of their data for research, had no symptoms/signs documented at presentation, had duplicate study records, or were untraceable through NHS Digital (Fig. 1).

\section{Data collection and management}

Research nurses or colorectal administrative assistants examined patient records and notes, including referral letters, and recorded demographic and clinical details on bespoke SIGGAR trial proformas, including age, sex, general practitioner (GP)-reported symptoms, route and urgency of referral, and planned diagnostic investigations. There were tick boxes for 'abdominal pain', 'anaemia', 'change in bowel habit $(\mathrm{ClBH})$ ', 'positive FOBT' (faecal occult blood test), 'rectal bleeding', and 'weight loss'. Free text fields were included to record additional symptoms/signs that were coded and classified, and characteristics of reported $\mathrm{CIBHs}$, classified as 'more frequent', 'less frequent', 'variable', or 'unspecified'. Blood count data, additional clinical features, diagnostic investigations performed, and diagnoses during the hospital episode were ascertained through hospital record review.

For patients with blood count data, anaemia status was determined according to the results of blood tests performed within 6 months before and up to 3 months after referral. We examined the prevalence of anaemia and iron-deficiency anaemia (IDA) using two anaemia definitions: haemoglobin $(\mathrm{Hb})<13 \mathrm{~g} / \mathrm{dL}$ in men and $<12 \mathrm{~g} / \mathrm{dL}$ in women (broad definition anaemia), used by the World Health Organisation; and $\mathrm{Hb}<11 \mathrm{~g} / \mathrm{dL}$ in men and $<10 \mathrm{~g} / \mathrm{dL}$ in women (narrow definition anaemia), as in the 2005 NICE referral guidelines. ${ }^{18,19}$ IDA was classified on the basis of microcytosis (mean red cell corpuscular volume [MCV] $<80 \mathrm{fL}$ ) or serum ferritin $<20 \mu \mathrm{g} / \mathrm{L}$. For patients without blood count data, we defined anaemia based on whether the investigation of anaemia was indicated as a reason for referral on the pro-forma.

CRC diagnoses occurring within 3 years of referral were obtained from cancer registries, via NHS Digital, and hospital records. CRC sites were defined by International Classification of Diseases, tenth revision codes: proximal cancer included C18.0-C18.5 (caecum to splenic flexure) and distal cancer included C18.6, C18.7, C19, C20, and C21 (descending colon to anus). CRC morphologies were defined by International Classification of Diseases for Oncology, second edition codes; we included codes related to invasive and in situ carcinomas $(8000 / 3,8010 / 3,8070 / 3$,
$8123 / 3,8140 / 2,8140 / 3,8144 / 3,8210 / 3,8261 / 2,8261 / 3,8263 / 2$, $8263 / 3,8480 / 3,8481 / 3,8490 / 3,8510 / 3$, and 8560/3).

\section{Statistical analysis}

The primary outcome was the yield of proximal vs. distal cancer within 3 years of referral by presenting symptom/sign. Yields were calculated as the number of patients with proximal or distal cancer divided by the number of patients with a particular symptom/sign, presented as percentages. Secondary outcomes included the number of needed whole-colon examinations (NNE) to detect one proximal vs. distal cancer by presenting symptom/sign, calculated by inverting the yield and presented with binomial exact $95 \%$ confidence intervals (Cls). For this calculation, we made the assumption that $\mathrm{WCI}$ has perfect sensitivity for detecting proximal and distal cancers.

We calculated the sensitivity of symptoms/signs for proximal and distal cancer and the proportion of patients with CRC who had proximal vs. distal cancer by presenting symptom/sign. We also calculated the proportion of CRCs that would have been missed if certain symptoms/signs were investigated by flexible sigmoidoscopy alone, with binomial exact $95 \%$ Cls, assuming that flexible sigmoidoscopy would have detected all distal cancers but not proximal cancers.

We estimated that, with a sample size of 8,484 patients, giving rise to an estimated 68 proximal and 421 distal cancers, we could estimate with sufficient precision the proportion of CRCs that would have been missed if certain symptoms/signs were investigated by flexible sigmoidoscopy alone. Data analyses were conducted using Stata/IC 13.1 (StataCorp. 2013. Stata Statistical Software: Release 13. College Station, TX: StataCorp LP).

\section{RESULTS}

A total of 8484 patients referred to hospital with suspected CRC were assessed for eligibility for the SIGGAR trials. Of these, 5448 were randomised to the SIGGAR trials and 3036 were not (Fig. 1). Comparing randomised and non-randomised patients, the former were younger and less likely to have been referred from a colorectal surgical outpatient clinic and via the urgent pathway (data not shown).

Of the total 8484 patients, all of whom were deemed eligible for the SOCCER study, 1109 were subsequently excluded, primarily due to patient dissent to use of their data for research. This left 7375 patients for our cohort analysis (Fig. 1). The median age of included patients was 69 years (interquartile range: 62-76) and $59.0 \%$ were women. The majority of patients were referred from a colorectal surgical outpatient clinic (84.5\%) and via the urgent pathway (71.7\%) (Table 1), and 1483 (20.1\%) had undergone flexible sigmoidoscopy at the time of referral (data not shown).

In total, 556 CRCs were diagnosed in 551 of the 7375 patients (7.5\%) within 3 years following referral (Table 2). Of the 551 patients with CRC, 522 (94.7\%) were diagnosed within 6 months post-referral (data not shown). There were 127 proximal and 429 distal cancers ( 5 patients had synchronous proximal and distal cancer), giving diagnostic yields of $1.7 \%$ for proximal cancer and $5.8 \%$ for distal cancer (Table 2). Detailed subsite information is presented in Supplementary Table 1.

Blood count data were not available for all patients. Data on $\mathrm{Hb}$ and MCV were available for 4742 of 7375 patients (64.3\%). There were 1157 patients $(15.7 \%)$ with serum ferritin in addition to $\mathrm{Hb}$ and MCV counts (Table 1). Comparing the 4742 patients with $\mathrm{Hb}$ and MCV data to the 2633 patients without, those with data available were older and less likely to have been referred from a colorectal outpatient clinic (Supplementary Table 2).

Among the 4742 patients with blood count data, narrow definition anaemia was present in $672(14.2 \%)$ and narrow definition anaemia with IDA was present in 363 (7.7\%). Broad definition anaemia was present in 1660 (35.0\%) and broad 
8484 referred with suspected CRC

5448 randomised in SIGGAR

3036 not randomised in SIGGAR

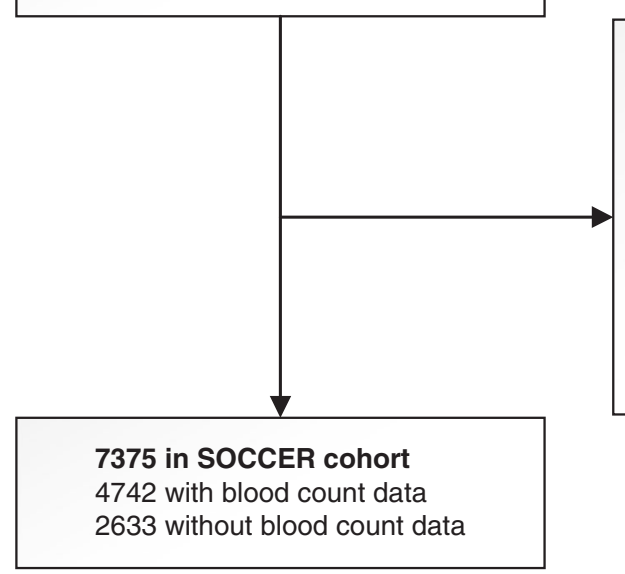

1109 excluded from SOCCER

936 dissented to use of data in

future research

75 judged unable to provide

informed consent

32 had no symptoms/signs recorded

at presentation

10 had duplicate study records

56 not traceable with NHS Digital

7375 in SOCCER cohort

4742 with blood count data

2633 without blood count data

Fig. 1 Flow chart of study participants CRC colorectal cancer, SIGGAR Special Interest Group in Gastrointestinal and Abdominal Radiology, SOCCER Symptoms of Colorectal Cancer Evaluation Research. The SOCCER study included patients who had been referred to hospital with suspected CRC and assessed for eligibility for the SIGGAR trials. All patients who met the SIGGAR trials eligibility criteria (regardless of whether they proceeded to randomisation) were eligible for the SOCCER study, unless they had dissented to use of their data in future research, were judged unable by a clinician to provide informed consent for the use of their data in future research, had no symptoms/signs recorded at presentation, had duplicate study records, or were not traceable with NHS Digital. Among those included in the SOCCER study, data on haemoglobin and mean red cell corpuscular volume were available for 4742 of the 7375 patients

\begin{tabular}{|c|c|c|}
\hline Characteristic & $N$ & $\%$ \\
\hline \multicolumn{3}{|l|}{ Sex } \\
\hline Men & 3023 & 41.0 \\
\hline Women & 4352 & 59.0 \\
\hline \multicolumn{3}{|l|}{ Age (years) } \\
\hline $55-64$ & 2407 & 32.6 \\
\hline $65-74$ & 2739 & 37.1 \\
\hline $75-84$ & 1896 & 25.7 \\
\hline$\geq 85$ & 333 & 4.5 \\
\hline \multicolumn{3}{|l|}{ Route of referral } \\
\hline Colorectal surgical outpatient clinic & 6231 & 84.5 \\
\hline Other outpatient clinic & 688 & 9.3 \\
\hline Straight to test & 396 & 5.4 \\
\hline Hospital admission & 33 & 0.4 \\
\hline Not recorded & 27 & 0.4 \\
\hline \multicolumn{3}{|l|}{ Urgency of referral } \\
\hline Urgent & 5290 & 71.7 \\
\hline Soon & 660 & 9.0 \\
\hline Routine & 914 & 12.4 \\
\hline Not recorded & 511 & 6.9 \\
\hline \multicolumn{3}{|l|}{ Availability of blood count data ${ }^{a}$} \\
\hline $\mathrm{Hb}$ and $\mathrm{MCV}$ & 4742 & 64.3 \\
\hline Serum ferritin & 1157 & 15.7 \\
\hline None available & 2633 & 35.7 \\
\hline
\end{tabular}

definition anaemia with IDA in 567 (12.0\%) (Table 2). The prevalence of anaemia increased with age among men and women. Broad definition anaemia was present in $23.8 \%(131 / 551)$ of men and in 18.6\% (161/867) of women aged 55-64 years and in $73.3 \%(66 / 90)$ of men and $57.2 \%(83 / 145)$ of women aged $\geq 85$ years. This pattern of anaemia increasing with age was also observed when considering the other anaemia definitions and IDA (data not shown). Among the 2633 patients without blood count data, investigation of anaemia was a reason for referral in 229 (8.7\%) (Table 2).

Anaemia and cancer site

Among the 4742 patients with blood count data, there were 97 proximal and 240 distal cancers. While yields of distal cancer did not vary by anaemia status, anaemia was strongly associated with proximal cancer (Table 2). Proximal cancer yield was $0.6 \%$ (19/ 3082 ) in patients without anaemia, increasing to $10.5 \%(38 / 363)$ in patients with narrow definition anaemia and IDA. Broadening the definition of anaemia and removing the requirement for IDA reduced the yield to $4.7 \%$ (78/1660). Among the 2633 patients without blood count data, yield of proximal cancer was 3.9\% (9/ $229)$ in patients referred for investigation of anaemia vs. $0.9 \%$ (21/ 2404) in those who were not (Table 2).

Although the yield of proximal cancer was highest in patients with narrow definition anaemia and IDA, this criterion identified $39.2 \%(38 / 97)$ of patients with proximal cancer, while the broad definition of anaemia (with or without IDA) identified 80.4\% (78/ 97) (Table 2). We therefore used the broad definition in all subsequent analyses of patients with blood count data $(n=4742)$ to ensure high sensitivity of anaemia for proximal cancer. For patients without blood count data $(n=2633)$, we defined anaemia based on whether the investigation of anaemia was indicated as a reason for referral. Among all 7375 patients, 1889 (25.6\%) had either broad definition anaemia $(n=1660)$ or were referred for investigation of anaemia $(n=229)$ (Table 2$)$. 


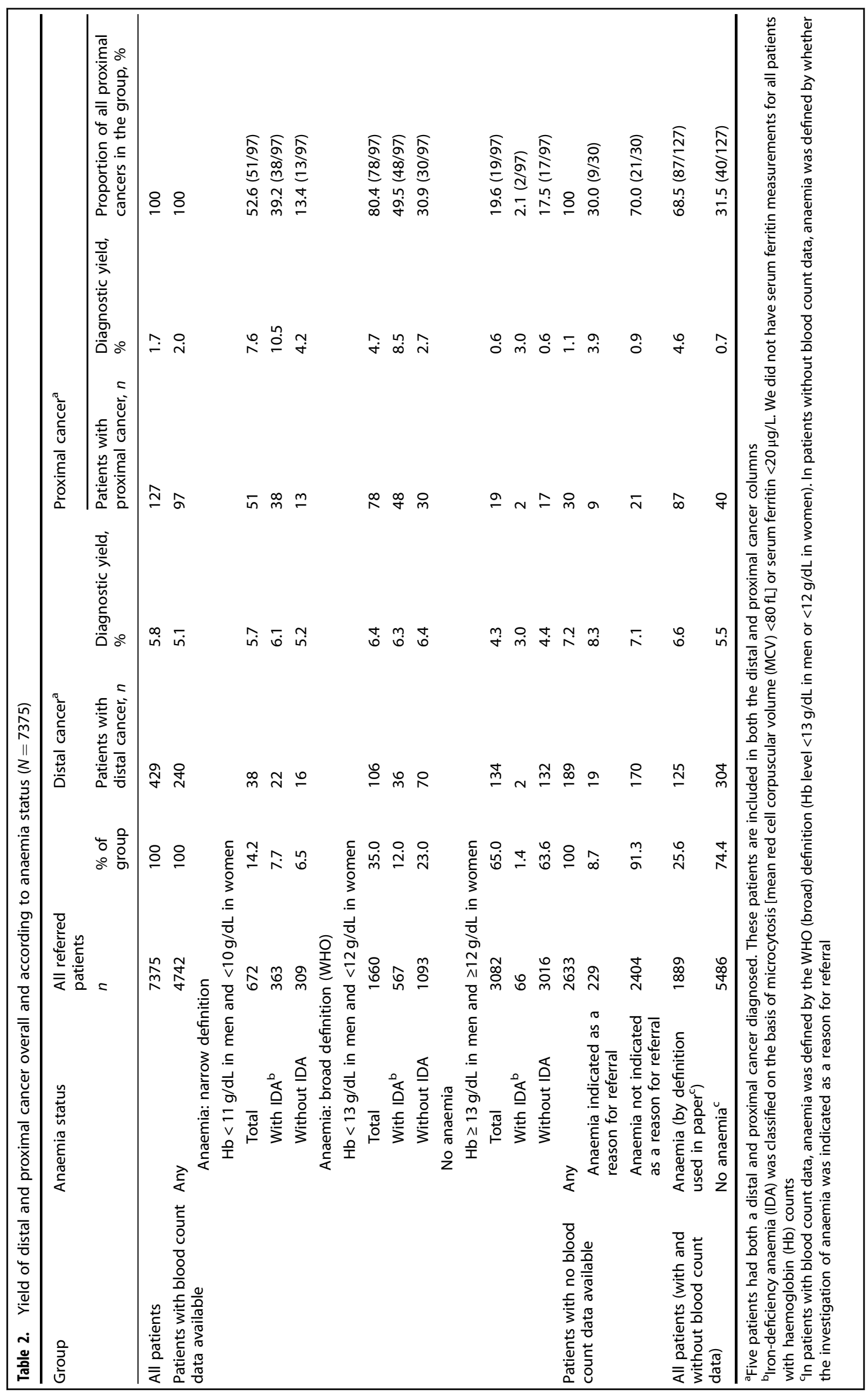




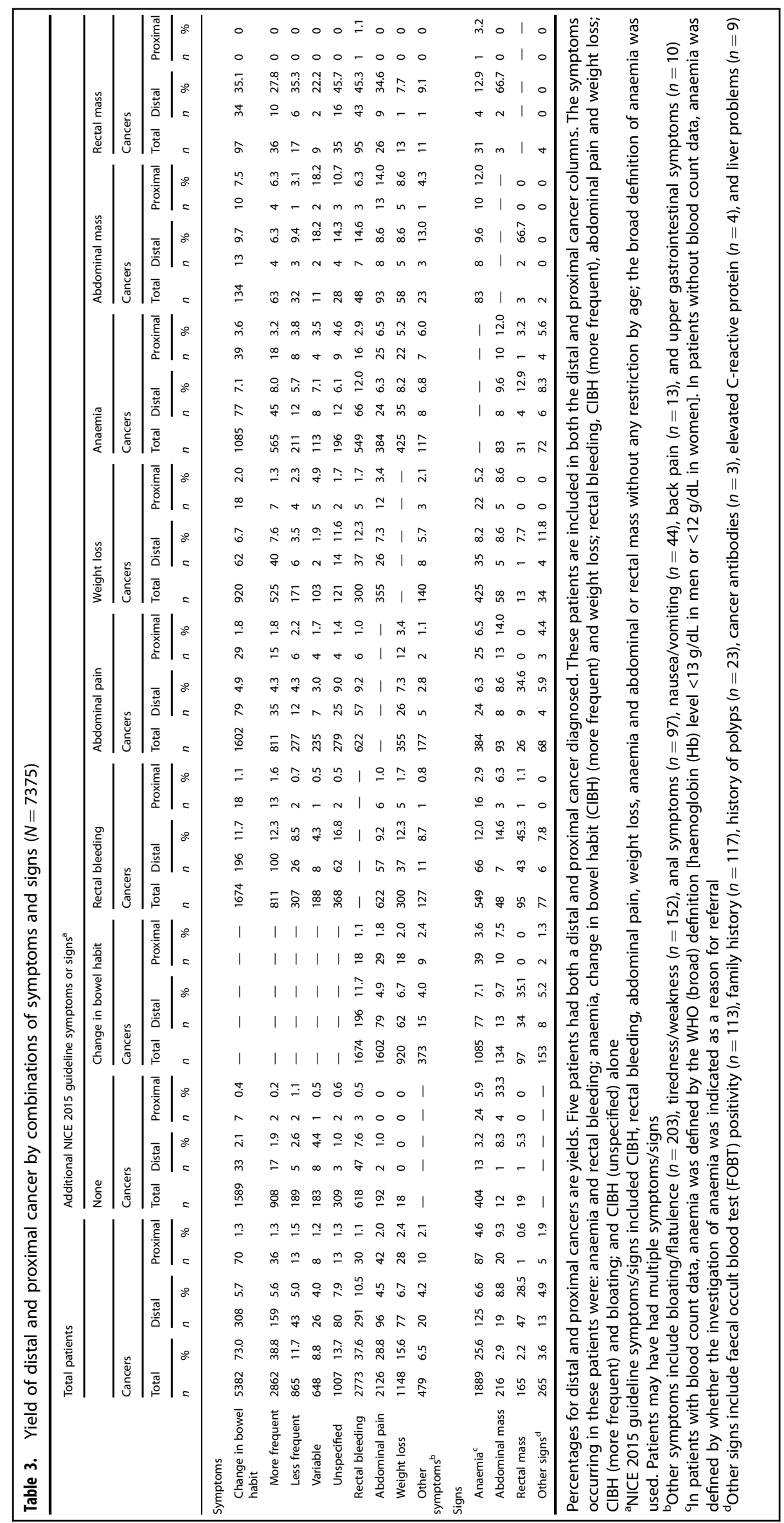




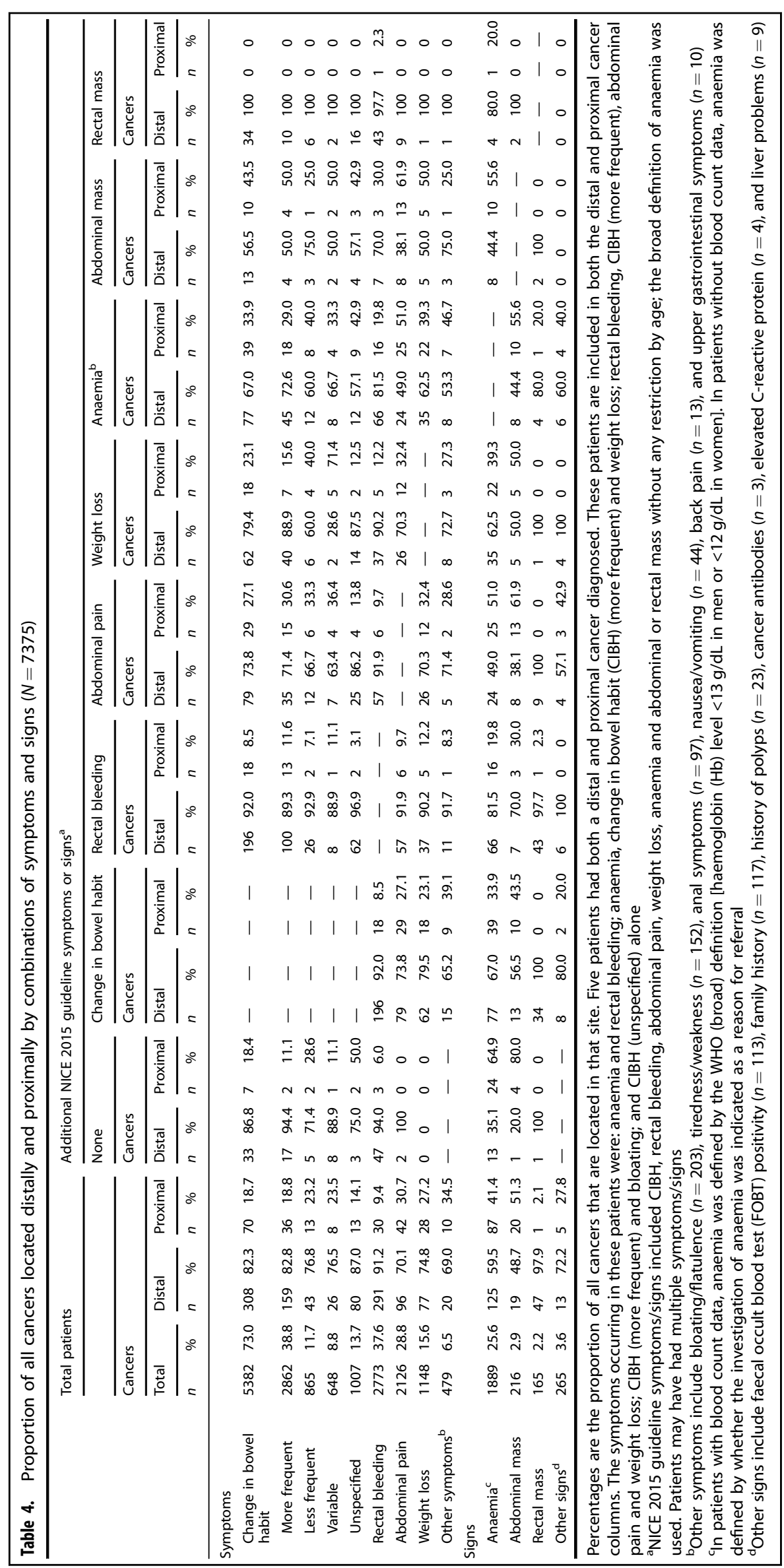




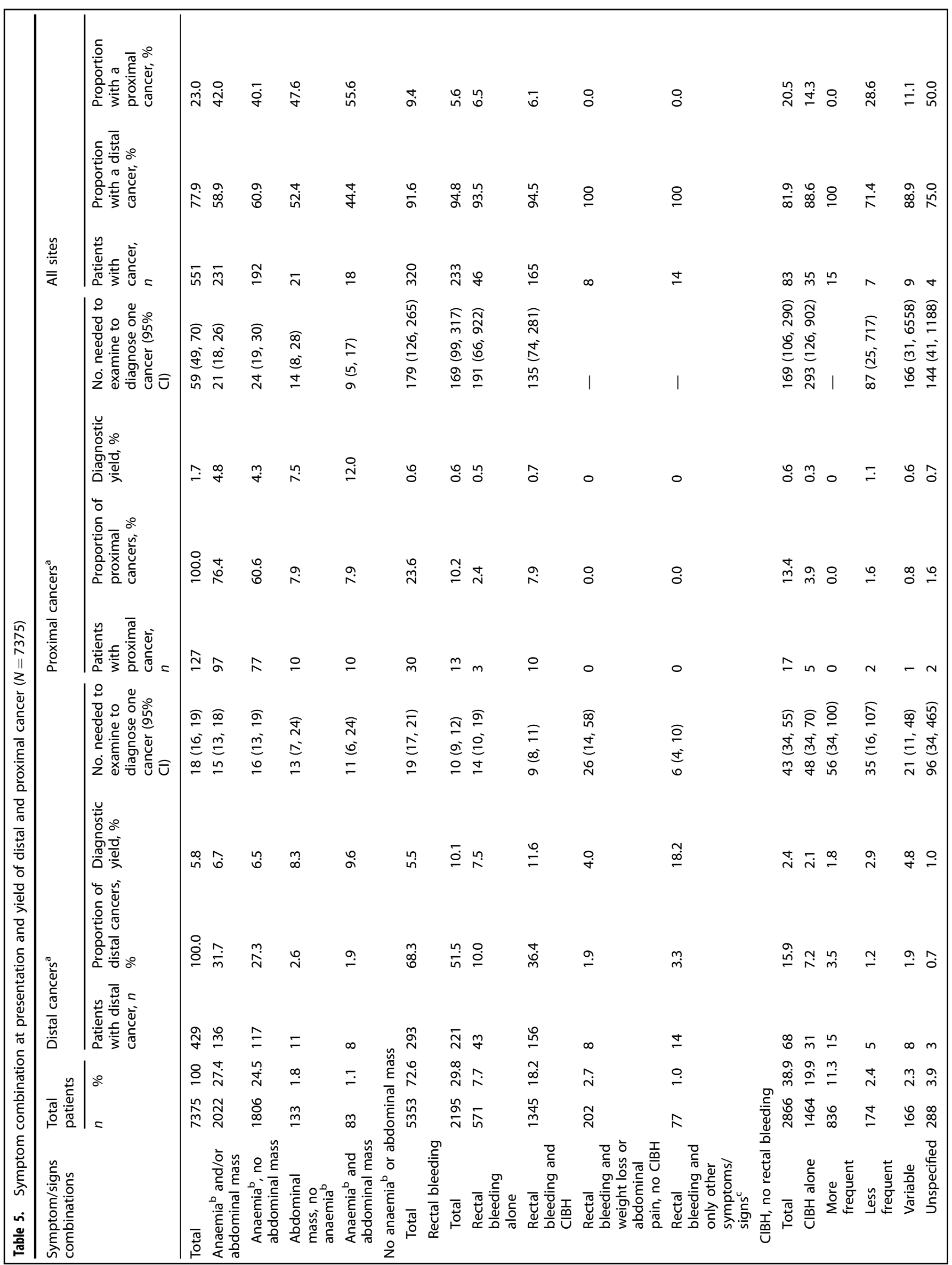


Whole-colon investigation vs. flexible sigmoidoscopy for suspected...

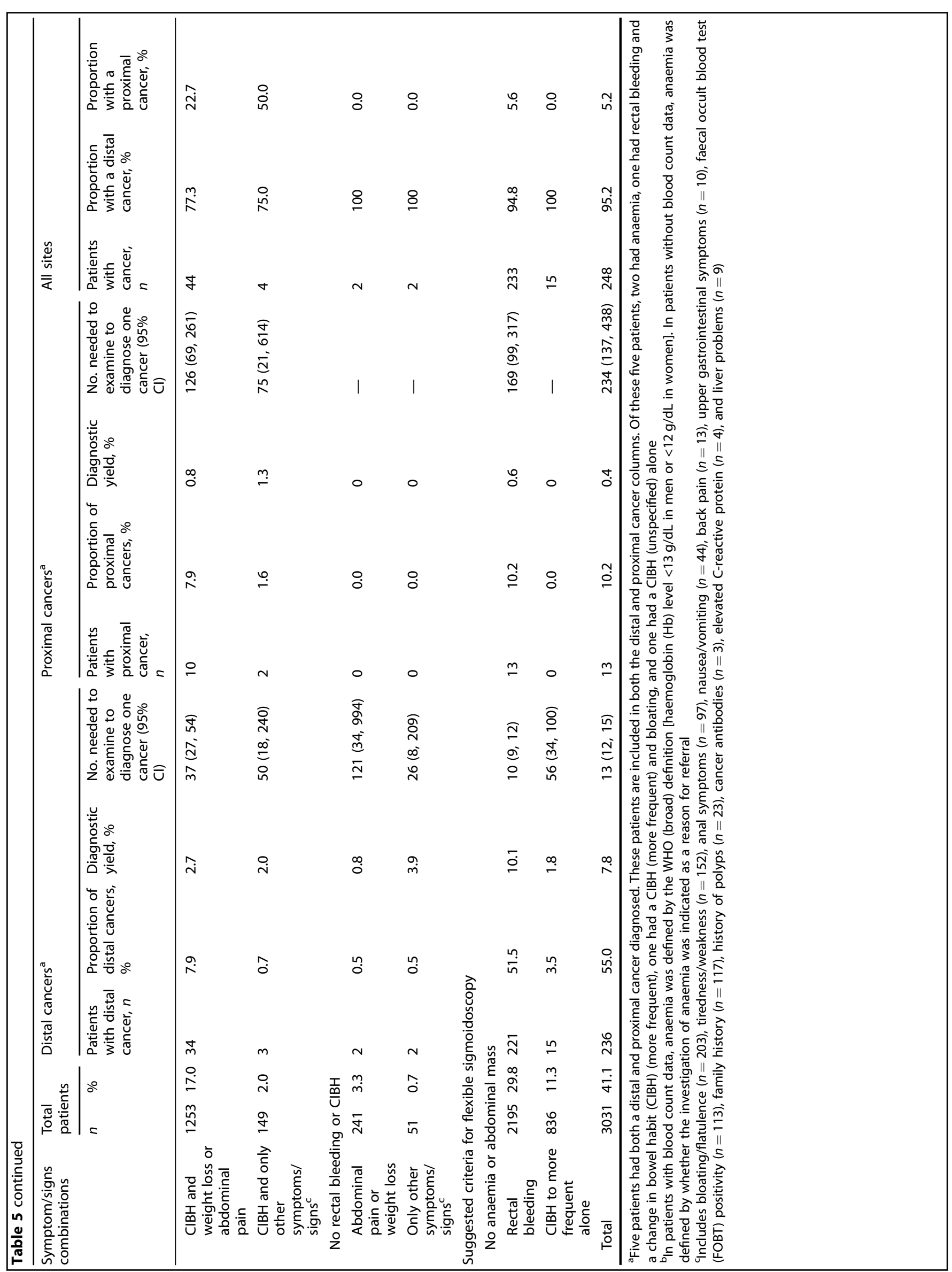


Symptoms and signs related to cancer site

Among all patients, the most common symptoms/signs were a $\mathrm{CIBH}(73.0 \%, n=5382)$, rectal bleeding $(37.6 \%, n=2773)$, abdominal pain $(28.8 \%, n=2126)$, weight loss $(15.6 \%, n=1148)$, and anaemia $(25.6 \%, n=1889)$ (Table 3$)$. Most patients presented with more than one symptom/sign (Supplementary Table 3).

Yields of proximal and distal cancer varied considerably by symptom/sign (Table 3). The highest yields of distal cancer were among patients with rectal mass $(28.5 \%, 47 / 165)$ and rectal bleeding $(10.5 \%, 291 / 2773)$. Yields of proximal cancer were generally lower than for distal cancer, with the highest yields among patients with abdominal mass $(9.3 \%, 20 / 216)$ and anaemia $(4.6 \%, 87 / 1889)$. Yields of proximal and distal cancer were generally higher when patients presented with a combination of symptoms/signs (Table 3).

The location of diagnosed cancers was also influenced by symptom/sign (Table 4). Rectal bleeding was associated with an approximate $90 \%$ chance that cancer was distal, irrespective of additional symptoms. Anaemia and abdominal mass were associated with a high probability that cancer was proximal $(41.4 \%$ and $51.3 \%$, respectively), irrespective of additional symptoms.

We evaluated the combination of anaemia and/or abdominal mass in further detail, given the strong association with proximal cancer. Among the 2022 patients with anaemia and/or abdominal mass, proximal cancer yield was $4.8 \%$, the NNE was $21(95 \% \mathrm{Cl}$ $18-26)$, and $42.0 \%$ of the patients with cancer had proximal cancer. The sensitivity of anaemia and/or abdominal mass for proximal cancer was $76.4 \%(97 / 127)$ (Table 5).

Among the 5353 patients without anaemia and/or abdominal mass, the yield of proximal cancer was $0.6 \%$ (NNE $179,95 \% \mathrm{Cl}$ 126-265). Proximal cancer yield was also $0.6 \%$ (NNE 169, $95 \% \mathrm{Cl}$ 99-317) among the 2195 patients without anaemia and/or abdominal mass who had rectal bleeding. No proximal cancers were found in 836 patients without anaemia and/or abdominal mass who presented with a $\mathrm{CIBH}$ to increased frequency alone (Table 5).

There were 3031 patients without anaemia and/or abdominal mass who had rectal bleeding or solely a $\mathrm{CIBH}$ to increased frequency, accounting for $41.1 \%$ of the cohort. Among these patients, 236 distal cancers were diagnosed (yield of 7.8\%; NNE 13, $95 \% \mathrm{Cl} 12-15)$ while only 13 proximal cancers were diagnosed (yield of $0.4 \%$; NNE 234, 95\% Cl 137-438) (Table 5). Yields of proximal cancer were $<1 \%$ for all age ranges in this patient subgroup (data not shown). Of the 13 proximal cancer patients, 6 had distal findings that would warrant WCI (Supplementary Table 4). Therefore, if this patient subgroup were investigated by flexible sigmoidoscopy alone, 7 of the total 556 cancers $(1.3 \%$, $95 \% \mathrm{Cl} 0.5-2.6 \%)$ would have been missed. For five of these seven cases, blood count data were not available and so anaemia at presentation cannot be ruled out.

Flexible sigmoidoscopy is considered complete if the sigmoid-descending colon junction is reached ${ }^{20}$; therefore, cancers in the descending colon may be missed. Descending colon cancers were rare in our study $(1.4 \%, 8 / 556)$. Of the eight patients with descending colon cancers, two had the symptom profile of rectal bleeding or solely a $\mathrm{CIBH}$ to increased frequency without anaemia and/or abdominal mass; one of these had a synchronous cancer in the sigmoid colon while the other had no important distal findings (Supplementary Table 5).

\section{DISCUSSION}

For patients referred to hospital with suspected CRC, an important decision is whether to offer WCI or flexible sigmoidoscopy. NHS clinics offering rapid access flexible sigmoidoscopy have been shown to be suitable for evaluation of urgently referred patients, with a low miss rate of proximal cancer., ${ }^{9,11}$ However, fear of missing proximal cancers remains, with $20-70 \%$ of patients proceeding to $\mathrm{WCI}$ following flexible sigmoidoscopy. $8,9,11,21$ This negates the convenience and cost-effectiveness of flexible sigmoidoscopy, particularly as subsequent $\mathrm{WCl}$ has a very low cancer yield. ${ }^{8,9}$ To address the fear of missing cancers in patients examined solely by flexible sigmoidoscopy, we sought to determine in which patients the yield of proximal cancer and the probability of missing proximal cancer is low.

This multicentre cohort study of 7375 patients referred with suspected CRC to 21 hospitals throughout England validates previous studies that showed how yields of proximal and distal cancer vary by presenting symptom/sign..$^{8-14}$ The largest previous study of 16,433 patients referred to hospital from 1986 to 2001 concluded that flexible sigmoidoscopy alone was sufficient for patients with a $\mathrm{ClBH}$, rectal bleeding, and/or abdominal pain but without IDA, abdominal mass, severe symptoms, or significant flexible sigmoidoscopy findings. This was based on the finding that proximal cancer was very rare $(<1 \%)$ in such patients. ${ }^{8}$ In an extension of this study, including data collected from 1986 to 2007, proximal cancer was again associated with IDA and abdominal mass but not with a CIBH or rectal bleeding. ${ }^{22}$

Our study confirms the strong association between anaemia and abdominal mass with proximal cancer and newly demonstrates the importance of adopting a broad definition of anaemia. We found that $80 \%$ of proximal cancer cases could be identified using the broad definition (with or without IDA), compared to $39 \%$ using the narrow definition with requirement for IDA. The broad definition should therefore be adopted when selecting patients for flexible sigmoidoscopy since there is a greater likelihood of missing proximal cancers with the narrower definition. Given the importance of anaemia as a marker for proximal cancer, we also recommend that all patients referred with suspected CRC have a full blood count, unless an emergency investigation is required.

Through closer examination of cancer yield by combinations of symptoms and signs, we were able to define the criteria for flexible sigmoidoscopy more specifically. Proximal cancer risk was particularly low in patients without anaemia and/or abdominal mass who presented with a $\mathrm{ClBH}$ to increased frequency alone. Similarly, very few proximal cancers were detected in patients without anaemia and/or abdominal mass who presented with rectal bleeding, alone or with other symptoms/signs. These novel findings led us to conclude that flexible sigmoidoscopy alone is sufficient for patients without . anaemia and/or abdominal mass who present with any rectal bleeding or solely a $\mathrm{CIBH}$ to increased frequency, unless there are significant distal findings that warrant WCI (i.e. large or multiple adenomas, IBD) or the examination is incomplete. Patients fitting these criteria could be reassured that their risk of proximal cancer is very low but that they should return to their GP if symptoms continue. In our study, $41 \%$ of patients fulfilled these criteria for flexible sigmoidoscopy alone.

The probability of missing a proximal cancer is an important consideration. In the study by Thompson et al., 131 proximal cancers were diagnosed. ${ }^{8}$ Of the 37 cases without anaemia and/or abdominal mass, 20 would have undergone subsequent $\mathrm{WCl}$ because of severe symptoms or significant flexible sigmoidoscopy findings. Thus 17 proximal cancers (13\%) would have been missed if only flexible sigmoidoscopy was undertaken. With our criteria for flexible sigmoidoscopy, 10 proximal cancers (8\%) would have been missed in the Thompson data, and probably fewer if our broad anaemia definition had been considered. Adoption of our flexible sigmoidoscopy criteria would therefore minimise the probability of missing proximal cancers.

There is a small risk that distal cancers could go undetected if flexible sigmoidoscopy is undertaken alone. While flexible sigmoidoscopy, in theory, can reach the splenic flexure, the descending colon sometimes goes unexamined as there are no 
anatomical markers that the splenic flexure has been reached. ${ }^{20,23}$ Few cancers are, however, located in the descending colon. ${ }^{24}$ In our study, 8 of the 429 distal cancers were in the descending colon and only 1 of the 8 patients would have undergone flexible sigmoidoscopy alone with our criteria.

There is also a small risk of missing cancers in the sigmoid colon with flexible sigmoidoscopy. Examinations failing to reach the sigmoid-descending colon junction are, however, typically deemed incomplete, ${ }^{20,23,25}$ prompting subsequent whole-colon completion examination..$^{8,9,21}$ It is worth noting that, although studies have reported high rates of incomplete examinations due to pain, faeces, or scope looping, these were based on flexible sigmoidoscopy performed over 10 years ago. ${ }^{23,25}$ There are now remedies to avoid these scenarios. Premixed $50 \%$ nitrous oxide and oxygen (Entonox, BOC Healthcare, Guildford, Surrey, UK) has been shown to be as effective as conventional sedation for colonoscopy and is used frequently for flexible sigmoidoscopy screening in the English Bowel Cancer Screening programme. Preparation for flexible sigmoidoscopy usually involves a single phosphate enema, ${ }^{6}$ although oral preparations are occasionally used. If the distal bowel is not adequately cleared by an initial enema, a second can be administered via the endoscope. This has proved highly effective in patients having inadequate bowel preparation at colonoscopy. ${ }^{27}$

Isolated proximal non-cancerous abnormalities could also be missed by undertaking flexible sigmoidoscopy alone. Few studies, however, have addressed this. ${ }^{28,29}$ One retrospective study of 1766 patients who had undergone colonoscopy for rectal bleeding found isolated proximal colitis in $26(1.5 \%)$ patients. ${ }^{28}$ Similarly, in a study of 625 patients aged $<50$ years with diarrhoea, $10(1.6 \%)$ had isolated proximal IBD or colitis. ${ }^{29}$ Microscopic colitis, a common cause of unexplained chronic diarrhoea, presents a particular challenge as it can only be diagnosed on colonic biopsy and the vast majority of patients who receive a diagnosis have macroscopically normal colons. ${ }^{30}$ Although limited, the current evidence indicates that, when microscopic colitis is present, it is present throughout the colon. ${ }^{31,32}$ It is therefore likely that distal biopsies taken during flexible sigmoidoscopy would be sufficient for diagnosis.

Our study has several strengths, including recruitment from multiple hospitals, detailed baseline patient information, and follow-up using cancer registries for 3 years post-referral. We are confident that the follow-up period was sufficiently long to capture all incident CRC cases as the vast majority of CRCs were diagnosed within 6 months, with very few diagnosed in the ensuing 3 years.

Limitations include a possible selection bias since all patients were aged $\geq 55$ years, considered fit for $\mathrm{WCl}$, and $72 \%$ were urgently referred. Some patients did not proceed to undergo $\mathrm{WCl}$ but we were unable to accurately identify these patients from hospital records; nevertheless, we are confident that we did not miss cancers because we had cancer registry data for 3 years post-referral. Reporting bias is possible as the recording of symptoms by medical staff may have been influenced by what investigations were planned or by the presumed significance of the symptoms. ${ }^{33}$

A further limitation is that we did not have blood count data on all patients; for those patients without this data, anaemia was defined by whether it was indicated as a reason for referral. With full blood count data, it is possible that even fewer proximal cancer cases would have been missed by our flexible sigmoidoscopy criteria, as a greater proportion may have had anaemia. Finally, it is important to note that the cohort was gathered before the Bowel Cancer Screening Programme was fully rolled out in England. It is now common in clinical practice to consider the screening history of patients along with how recently any colonic examinations have taken place when selecting the most appropriate diagnostic investigation.

\section{CONCLUSION}

Our findings confirm the strong association between anaemia and proximal cancer, with $80 \%$ of proximal cancer patients in our cohort meeting the criteria for broad definition anaemia. All patients with suspected CRC should therefore have a full blood count, unless an emergency investigation is needed, and patients identified as anaemic according to the broad definition of anaemia should be referred for WCl. Conversely, the risk of proximal cancer is very low in patients without broad definition anaemia and/or abdominal mass but who present with any rectal bleeding or solely a CIBH to increased frequency. Patients with this symptom profile can be examined safely by flexible sigmoidoscopy alone. If incorporated into guidelines, this strategy would greatly reduce the number of WCls performed in patients at low risk of proximal cancer; this would alleviate the burden of $\mathrm{WCl}$ on patients and endoscopy and radiology services.

\section{ACKNOWLEDGEMENTS}

The investigators are grateful to the people listed below for their involvement in this study. In addition to those named, we would like to thank all of the personnel involved in the SIGGAR trials. CSPRG study staff: Mrs Elizabeth Coles (research assistance); Dr Paula Kirby (project management); Dr Fiona Lucas (literature review and editorial assistance); Miss Bhavita Patel (data collection, acquisition, cleaning and coding); Mr lain Stenson (data collection and cleaning and information governance); and Dr Laura Turner (information governance, ethical approvals and project management). SOCCER steering committee: Professor Greg Rubin - Chair, independent (professor of general practice and primary care, University of Durham); Mr Omar Faiz - Non-independent (consultant colorectal surgeon, Imperial College London); Dr Pawan Randev - Independent (GP/primary care lead, London Cancer Alliance); Dr John De Caestecker - Independent (consultant gastroenterologist, Leicester General); Professor Richard Logan - Independent (professor of epidemiology/consultant gastroenterologist, University of Nottingham); Helen Watson - patient representative, Independent (arranged through Bowel Cancer UK). A special thank you to all of the patients who contributed data to the study.

\section{FUNDING}

This is a summary of independent research funded by the National Institute for Health Research (NIHR) Health Technology Assessment Programme and the Bobby Moore Fund for Cancer Research UK. The funders had no involvement in study design, data collection, data analysis, manuscript preparation, or publication decisions. The views expressed are those of the authors and not necessarily those of the NHS, the NIHR, the Department of Health or Cancer Research UK. Infrastructure support for this work was provided by the NIHR Imperial Biomedical Research Centre (BRC).

\section{AUTHOR CONTRIBUTIONS}

W.A., W.H., and S.H. were responsible for study design. A.J.C. and W.A. were responsible for oversight of data analysis. K.P. acquired supplemental raw data K.W. did the statistical analyses and A.J.C., W.A., and K.W. were responsible for data interpretation. E.C.R., J.P.B., M.R.T., S.H., S.T.-G., and B.P.S. critically appraised the data. A.J.C., W.A., K.W., E.C.R., and J.P.B. drafted the manuscript. K.P.,W.H., M.R.T., K.G.F., S.H., S.T.-G., M.V., and B.P.S. critically appraised the final manuscript. All authors gave final approval on the version to be published.

\section{ADDITIONAL INFORMATION}

Supplementary information is available for this paper at https://doi.org/10.1038/ s41416-018-0335-z.

Availability of data and materials: Because of the sensitive nature of the data collected for this study, requests to access the data set from qualified researchers trained in human subject confidentiality protocols may be sent to the corresponding author.

Ethics approval and consent to participate: The SIGGAR trials were registered (ISRCTN95152621) and received ethics approval from the Northern and Yorkshire Multicentre Research Ethics Committee (MREC/3/3/075). Written informed consent was obtained from all patients randomised in the SIGGAR trials. Permission to obtain cancer and mortality data for patients not randomised in the SIGGAR trials was 
granted by the National Information Governance Board (ref. ECC 5-04(E)/2011). Further ethics approval for the SOCCER study was granted by the National Research Ethics Service (NRES) Committee North East - York. Approval for the processing of patient identifiable information without consent under Section 251 of the NHS Act 2006 was granted by the NHS Health Research Authority Confidentiality Advisory Group (ref. 14/CAG/1043).

Competing interests: S.T.-G. reports fees from PENTAX Medical and Olympus for equipment loans and from Fujifilm and Aquilant for educational course sponsorship and support. The other authors declare no competing interests.

\section{REFERENCES}

1. National Institute for Health and Clinical Excellence. Colorectal Cancer: Diagnosis and Management. NICE Guidelines (CG131). (Last Update 2014) 1-25 (NICE, London, 2011).

2. National Institute for Health and Care Excellence. Suspected Cancer: Recognition and Referral. NICE Guidelines (NG12). (Last Update. 2017) 1-79 (NICE, London, 2015).

3. Brown, H. et al. Scoping the Future: An Evaluation of Endoscopy Capacity Across the NHS in England 1-90 (Cancer Research UK, London, UK, 2015).

4. Bending, M. W. et al. Estimating the direct costs of bowel cancer services provided by the National Health Service in England. Int. J. Technol. Assess. Health Care 26, 362-369 (2010).

5. Lawler, M. et al. Critical research gaps and recommendations to inform research prioritisation for more effective prevention and improved outcomes in colorectal cancer. Gut 67, 179-193 (2018).

6. Atkin, W. S. et al. Single blind, randomised trial of efficacy and acceptability of oral picolax versus self administered phosphate enema in bowel preparation for flexible sigmoidoscopy screening. BMJ 320, 1504-1508 (2000).

7. Maslekar, S., Hughes, M., Gardiner, A., Monson, J. R. \& Duthie, G. S. Patient satisfaction with lower gastrointestinal endoscopy: doctors, nurse and nonmedical endoscopists. Colorectal Dis. 12, 1033-1038 (2010).

8. Thompson, M. R. et al. Flexible sigmoidoscopy and whole colonic imaging in the diagnosis of cancer in patients with colorectal symptoms. Br. J. Surg. 95, 1140-1146 (2008).

9. Royle, T. J. et al. Same-day assessment and management of urgent (2-week wait) colorectal referrals: an analysis of the outcome of 1606 patients attending an endoscopy unit-based colorectal clinic. Colorectal Dis. 16, O176-0181 (2014).

10. Couch, D. G., Murphy, J. H., Boyle, K. M. \& Hemingway, D. M. Straight to flexible sigmoidoscopy: rationalization of 2-week wait referrals in suspected colorectal cancer. Colorectal Dis. 17, 980-983 (2015).

11. Badiani, S., Desai, A. \& Chapman, M. A. Is whole colonic imaging necessary for symptoms of change in bowel habit and/or rectal bleeding? Colorectal Dis. 14, 1197-1200 (2012).

12. Kent, A. J., Woolf, D., McCue, J. \& Greenfield, S. M. The use of symptoms to predict colorectal cancer site. Can we reduce the pressure on our endoscopy services? Colorectal Dis. 12, 114-118 (2010).

13. Bhangu, A. et al. Detection and survival of colorectal cancer from a 2 week wait service. Surgeon 9, 78-82 (2011).

14. Macdonald, S., Radhakrishnan, S. \& Seward, E. Is flexible sigmoidoscopy ever enough? An audit of the rates of proximal disease during colonoscopy. Gut 62 (Suppl 1(abstract)), A222-A223 (2013).

15. Atkin, W. et al. Is whole-colon investigation by colonoscopy, computerised tomography colonography or barium enema necessary for all patients with colorectal cancer symptoms, and for which patients would flexible sigmoidoscopy suffice? A retrospective cohort study. Health Technol. Assess. 21, 1-80 (2017).

16. Halligan, S. et al. Computed tomographic colonography versus barium enema for diagnosis of colorectal cancer or large polyps in symptomatic patients (SIGGAR): a multicentre randomised trial. Lancet 381, 1185-1193 (2013).
17. Atkin, W. et al. Computed tomographic colonography versus colonoscopy for investigation of patients with symptoms suggestive of colorectal cancer (SIGGAR): a multicentre randomised trial. Lancet 381, 1194-1202 (2013).

18. World Health Organisation. Haemoglobin Concentrations for the Diagnosis of Anaemia and Assessment of Severity 1-6 (World Health Organisation, Geneva, Switzerland, 2011)

19. National Institute for Health and Clinical Excellence. Referral Guidelines for Suspected Cancer. NICE Guidelines (CG27) 1-98 (NICE, London, 2005).

20. Lehman, G. A., Buchner, D. M. \& Lappas, J. C. Anatomical extent of fiberoptic sigmoidoscopy. Gastroenterology 84, 803-808 (1983).

21. Pullens, H. J., Joosten, M., Siersema, P. D. \& Brink, M. A. Open-access flexible sigmoidoscopy frequently leads to additional colonoscopy in symptomatic patients over 50 years. J. Gastrointestin. Liver. Dis. 23, 153-159 (2014).

22. Thompson, M. R. et al. Clinical assessment to determine the risk of bowel cancer using Symptoms, Age, Mass and Iron deficiency anaemia (SAMI). Br. J. Surg. 104, 1393-1404 (2017).

23. Painter, J. et al. Depth of insertion at flexible sigmoidoscopy: implications for colorectal cancer screening and instrument design. Endoscopy 31, 227-231 (1999).

24. Cancer Research UK. Bowel cancer incidence statistics. [Available from: http:// www.cancerresearchuk.org/health-professional/cancer-statistics/statistics-bycancer-type/bowel-cancer/incidence (accessed August 2018).

25. Eloubeidi, M. A., Wallace, M. B., Desmond, R. \& Farraye, F. A. Female gender and other factors predictive of a limited screening flexible sigmoidoscopy examination for colorectal cancer. Am. J. Gastroenterol. 98, 1634-1639 (2003).

26. NHS England. NHS Public Health Functions Agreement 2017-18. Service Specification No. 26A NHS Bowel Scope Screening Programme 1-38 (NHS England, London, UK, 2017).

27. Sim, J. S. \& Koo, J. S. Predictors of inadequate bowel preparation and salvage options on colonoscopy. Clin. Endosc. 49, 346-349 (2016).

28. Mulcahy, H. E. et al. Yield of colonoscopy in patients with nonacute rectal bleeding: a multicenter database study of 1766 patients. Am. J. Gastroenterol. 97, 328-333 (2002).

29. Shale, M. J., Walters, J. R. \& Westaby, D. Adequacy of flexible sigmoidoscopy with biopsy for diarrhea in patients under age 50 without features of proximal disease. Gastrointest. Endosc. 73, 757-764 (2011).

30. Storr, M. A. Microscopic colitis: epidemiology, pathophysiology, diagnosis and current management-an update 2013. ISRN Gastroenterol. 2013, 352718 (2013).

31. Genta, R. M. \& Sonnenberg, A. The yield of colonic biopsy in the evaluation of chronic unexplained diarrhea. Eur. J. Gastroenterol. Hepatol. 27, 963-967 (2015).

32. Macaigne, G. et al. Microscopic colitis or functional bowel disease with diarrhea: a French prospective multicenter study. Am. J. Gastroenterol. 109, 1461-1470 (2014).

33. Price, S. J., Stapley, S. A., Shephard, E., Barraclough, K. \& Hamilton, W. T. Is omission of free text records a possible source of data loss and bias in Clinical Practice Research Datalink studies? A case-control study. BMJ Open 6, e011664 (2016).

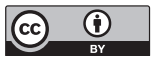

Open Access This article is licensed under a Creative Commons Attribution 4.0 International License, which permits use, sharing, adaptation, distribution and reproduction in any medium or format, as long as you give appropriate credit to the original author(s) and the source, provide a link to the Creative Commons license, and indicate if changes were made. The images or other third party material in this article are included in the article's Creative Commons license, unless indicated otherwise in a credit line to the material. If material is not included in the article's Creative Commons license and your intended use is not permitted by statutory regulation or exceeds the permitted use, you will need to obtain permission directly from the copyright holder. To view a copy of this license, visit http://creativecommons. org/licenses/by/4.0/.

(c) The Author(s) 2018 